\title{
CIRCULATORY STUDIES OF THE FUNDUS OF THE EYE*
}

\author{
BY \\ P. WEINSTEIN and J. Forgács \\ BUDAPEST
}

OUR investigations assumed first that the difference of pressure between the arterial and venous parts of the capillary system acts as circulatory motor, further, that spontaneous venous pulsation of the fundus of the eye is a sign of a pressure prevailing within the central retinal vein lower than the intra-ocular tension. One hundred cases were examined: fifty of them showing spontaneous pulsation, the other fifty without such. Hardly any difference concerning number of hypertonics and hypotonics, ocular tension, age, sex and brachial blood-pressure could be distinguished between the two groups in question (see Table). With those cases in which no

TABLE I

\begin{tabular}{|c|c|c|c|c|c|c|}
\hline & & & & & $\underset{50}{\text { ulsating cases }}$ & $\begin{array}{c}\text { Non-pulsating } \\
\text { cases } 50\end{array}$ \\
\hline Hypertonia & ... & $\ldots$ & $\ldots$ & $\ldots$ & 15 & 15 \\
\hline Hypotonia & $\ldots$ & $\ldots$ & $\ldots$ & $\ldots$ & 7 & 6 \\
\hline Ocular tension & $\ldots$ & $\ldots$ & $\ldots$ & $\ldots$ & $15-26$ & $13-23$ \\
\hline Age & $\ldots$ & $\cdots$ & $\ldots$ & $\ldots$ & $16-79$ & $16-76$ \\
\hline$\ldots$ & $\ldots$ & $\cdots$ & $\ldots$ & $\ldots$ & $\begin{array}{c}25-25 \\
8 \quad q\end{array}$ & $\begin{array}{c}31-19 \\
8 \&\end{array}$ \\
\hline \multirow{3}{*}{\multicolumn{5}{|c|}{$\begin{array}{l}\text { Blood-pressure } \ldots \\
\text { Appearance of arterial pulsation (mm. } \\
\text { mercury suction) } \ldots \text {. } \ldots \text {. } \\
\text { Appearance of venous pulsation (mm. }\end{array}$}} & $164 / 92$ & $160 / 90$ \\
\hline & & & & & 97 & 141 \\
\hline & & & & & 91 & 141 \\
\hline \multicolumn{5}{|c|}{$\begin{array}{ccc}\text { mercury suction }) \quad \ldots \quad \ldots & \ldots\end{array}$} & .27 & 59 \\
\hline \multirow{4}{*}{\multicolumn{3}{|c|}{$\begin{array}{l}\text { Retinal haemorrhage } \ldots \\
\text { Chorio-retinitis ... } \\
\text { Papilloedema } \ldots \\
\text { Atrophy of the optic nerve }\end{array}$}} & $\ldots$ & & 2 & 6 \\
\hline & & & $\cdots$ & $\begin{array}{l}\cdots \\
\ldots\end{array}$ & 2 & 4 \\
\hline & & & $\begin{array}{l}\cdots \\
\ldots\end{array}$ & $\cdots$ & 1 & 2 \\
\hline & & & $\ldots$ & $\ldots$ & 1 & - \\
\hline & & & & otal & $\begin{array}{l}6(12 \text { per } \\
\text { cent.) }\end{array}$ & $\begin{array}{l}12(24 \text { per } \\
\text { cent.) }\end{array}$ \\
\hline
\end{tabular}

spontaneous venous pulsation was present, such was elicited by applying Kukan's ophthalmo-dynamometer. We did not transpose the mercury millimetre values at the moment pulsation becomes visible, into values of retinal blood pressure, but in the table only

\footnotetext{
* Received for publication, January 13, $1947^{\circ}$
} 
that mercury millimetre value of suction is represented, at which venous or arterial pulsation becomes first evident.

The difference of the two groups manifests itself in two directions the drop from arterial and venous diastolic pressure established at a higher level with the non-pulsating cases, than with the pulsating ones (see Fig. I), although the value is the same, respectively the difference between arterial and venous pressure is essentially larger with the pulsating eye, The following has to be considered in

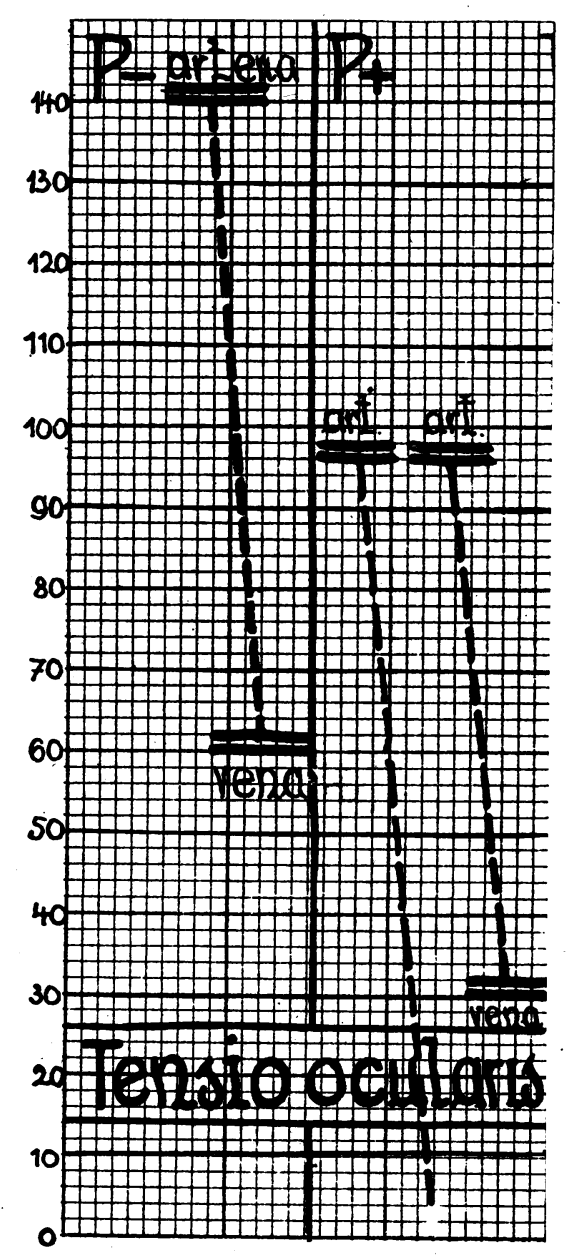

FIG. 1.

Suction-pressure exerted on the bulb of the eye by oph thalmodynamometer in $\mathrm{mm}$. of mercury, which elicited the first arterial or venous pulsation respectively. $\mathrm{P}-$ : non-pulsating cases, $\mathrm{P}+$ : spontaneous pulsating cases. 
evaluating these data: Kukan explains his method so that when he exerts suction on the sclera by the disk, the rim of it presses the bulb of the eye in the same ratio, and thereby the intra-ocular pressure is increased in such a degree, that it surpasses the pressure prevalent within the vessels and thus causes pulsation within the same. Lately Dubois and Fischer from Weve'siclinic (Ophthalmologica, 1941), proved that the intra-ocular pressure is not increased by this suction method, but the stretching of the sclera changes and the pressure of the retinal vessels decreases below the intra-ocular pressure thereby, and then pulsation ensues in them.

The other difference between the two groups is, that with nonpulsating cases the incidence of ophthalmological complications is 24 per cent. while in pulsating cases it is 12 per cent.

The difference between the two groups, namely, that the drop of pressure with pulsating cases is greater between the arterial and venous part of the retinal circulatory system than with the nonpulsating, and that the entire pressure-level of the latter is shifted higher and that incidence of retinal complications is only half with pulsating eyes as with non-pulsating, each follows from the other. The fact that arterial pressure is established at a higher level signifies that an obstacle prevails with the capillaries, to overcome which the pressure of the central retinal artery is increased. If again pressure is higher within the vein, that means worse circulatory conditions than when spontaneous pulsation prevails, because then the drop of pressure between artery and vein is higher and therefore circulation is more continuous. The following cases serve as instances:

1. Plesh curve of very large amplitude, high blood-pressure, spontaneous retinal venous pulsation absent, severe alterations of the fundus, nephrosclerosis.

2. A curve completely similar to the first, similarly increased high blood-pressure, spontaneous pulsation, intact fundus.

3. Curve of large amplitude, high blood-pressure, spontaneous pulsation, fundus haemorrhage and retinitis foci, complete restitution.

The presence of spontaneous venous pulsation with the two latter cases evidently signifies satisfactory capillary circulation and secures retinal supply and disposition for improvement, while with the first case despite high blood-pressure the circulation of the fundus is bad, because the level of the venous pressure is also increased, thereby the terminal points of the pressure-drop converging, the circulation becomes imperfect.

What about hypotonic cases established at a lower level of pressure? As long as the terminal points of the circulatory system show a difference of physiological pressure-drop, which keeps intact circulation going, this latter functions without trouble. But if this drop of pressure decreases, be it by further decrease of arterial pressure or increase of venous, the circulation becomes worse, as with those 
hypotonic cases showing spontaneous venous pulsation because this augments drop of pressure. A'ccording to Lauber and Sobansky ocular tension is significant with such hypotonic individuals regarding retinal circulation its relative increase already inhibiting retinal blood circulation. Our own investigations have not demonstrated any difference between hypertonics and hypotonics, pulsating and non-pulsating patients regarding ocular tension. According to Nakayima's experiments, persons using steam baths show a decrease of brachial diastolic pressure without exception, on account of the dilatation of the peripherial small vessels, similarly the diastolic pressure of the central retinal artery decreases, so that spontaneous arterial pulsation ensues and in the majority of cases even venous pulsation becomes evident, the system establishing a lower level of pressure, but ocular tension does not change in the course of the whole process. Normal ocular pressure evidently proceeds parallel to sudden increase or decrease of blood pressure, but not in ratio to continuously developing slow alteration of pressure.

Regarding the peculiarities of spontaneous venous pulsation or the artificially elicited one, venous pulsation elicited by pressure is characterized as already described by Fritz, by sudden increase and stop, according to Fritz a sign of satisfactory capillary permeability and suitable flow, or it increases gradually and slowly.

Or pulsation shows a fine vibration during the whole time, a transition towards that group, where no venous pulsation can be elicited because either capillary circulation is bad or diastolic pressure of the veins increased. Elicited diastolic pulsation of the vein appeared always preceding diastolic pulsation of the artery, and it subsided usually near arterial systolic pulsation ceasing, exceptionally near the cessation of diastolic pulsation.

Spontaneous venous pulsation occurs in 40 per cent. on both sides, 28 per cent. on the right and 32 per cent. on the left eye. Its origin is due, according to Serr, to undulations of the ocular tension synchronous with the revolutions of the heart, ocular tension surpassing venous pressure during systole, and causing compression of the vein. This phenomenon was very clearly visible in cases when the central retinal artery pulsated also and the pulsation of the central retinal vein had not yet ceased : the collapse of the vein exactly coincided with the systolic dilatation of the artery. Of course under pathological conditions there is the possibility of a penetrant-venous pulse originating. (Aortic ins., Basedow's disease, cranial tumour). In 10 per cent. of the cases spontaneous pulsation disappears if the patient lays down, as a proof that by.increase of intracranial pressure it increases within the vein also and thus surpassing intra-ocular pressure the pulsation ceases, as has been described by one of us already, 1938. (Weinstein : Brit. Jl. Ophthal, 1939, p. 396). In 18 per cent. of the cases besides the pulsation synchronous with the 
cardiac revolutions, a second separate rhythm of the vein, a formal allorhythmia is discernible, consisting of a ceasing of the rhythmic pulsation for seconds, frequently 4 to 5 then re-appearing again and being replaced by the original rhythm. Then after shorter or longer intervals, a pause again follows. This phenomenon (pulsus intermittens venosus retinae, Fig. 2), has not been described in human

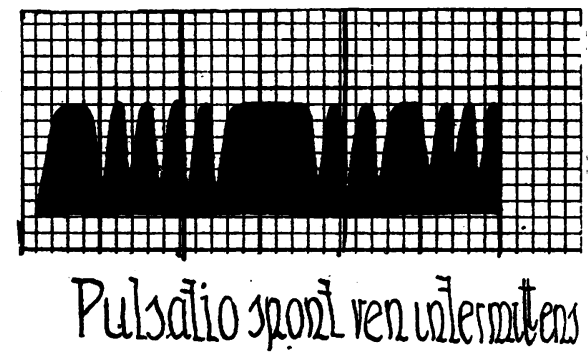

FIG. 2.

Pulsus intermittens spontaneous venosus retinae

ophthalmological physiology and pathology, it has been observed only in dogs as irregularly appearing temporary pulsation and was explained by undulations of tone in the blood-vessels. This intermittent pulsation observed by us, is probably also related to changes of tonicity of the capillaries and is apparent chiefly with vaso-neurotic individuals (migraine, epilepsy). As a practical consideraticn it follows, that investigating spontaneous venous pulsation the patient should be in a sitting position and the observation should be extended to at least one minute, observing the central retinal vein, because in the prostrate position pulsation frequently it is not visible and often ceases for seconds. With all our cases spontaneous venous pulsation disappeared if the eye was massaged, because then the ocular tension ceased and sank below venous pressure, whereby pulsation ceased for a time. (Seidel and Serr).

\section{Summary}

Individuals showing spontaneous venous pulsation have a higher drop of pressure between arterial and venous systems of retinal circulation compared with persons without pulsation, and consequently retinal circulation of the former is more satisfactory.

The level of arterial and venous diastolic pressure values of the. fundus of individuals showing no spontaneous venous pulsation are established at a higher level than those of the pulsating group, a circumstance pointing to a circulation inhibited to a high degree within the capillaries. 
Retinal complications show an incidence twice as high with cases without pulsation as with those pulsating.

Spontaneous venous pulsation disappears in 10 per cent. of the cases if the patient is laying down, and is of intermittent character in 18 per cent.

Spontaneous venous pulsation disappears if the bulb of the eye is massaged.

\section{BIBLIOGRAPHY}

Bailliart-La circulation retinienne, Paris, 1923.

DUKE-Elder-Diseases of the Inner Eye. Text-book of Ophthal., Vol. III, 1945. Orsalessi e Cassuto-La tonoscopia retinica. Firenze, 1938.

XV. Cong. Ophthal. 1937. Egypte. L'Hypertension arterielle de la retine.

\section{ANNOTATIONS}

\section{Moorfields, Westminster and Central Eye Hospital and The Ophthalmic Institute (University of London)}

The amalgamation of these three hospitals marks the beginning of a new era in the development of hospital services for eye disease in London. Implicit in this development lies the certainty of greatly improving the teaching and training of ophthalmologists and also the possibility of intensive research into the many unsolved problems of ophthalmology.

More than twenty years ago a scheme for the amalgamation of Moorfields and the Central Eye Hospital was discussed. Some of the preliminary work was done but the scheme failed because it was not thought possible to raise the necessarily large amount of money that would have been required to construct an entirely new hospital with the facilities and equipment then envisaged. This was all the more regrettable as at that time a vacant site adjacent to the Central was available which would have been amply large enough for the projected hospital and still leave space over for such development as the future might require.

Once this scheme was abandoned Moorfields proceeded to reconstruct itself on the very cramped site and the Central-then in a completely new building-just carried on. But in reality neither hospital was quite happy. Moorfields remained cramped and the Central soon found that its new building was not adequate to accommodate the new types of apparatus and the new departments which the developments of the science and practice of ophthalmology made necessary. One has only to mention such things as the slitlamp, orthoptics, light and X-ray treatment to show what expansion was necessary. A further problem was the training of ophthalmologists. Post-graduate teaching of ophthalmology became an 